Article

\title{
Graphene Aerogel Growth on Functionalized Carbon Fibers
}

\author{
Katerina Vrettos ${ }^{1}$, Konstantinos Spyrou ${ }^{2}$ and Vasilios Georgakilas ${ }^{1, *(D)}$ \\ 1 Department of Material Science, University of Patras, 26504 Patras, Greece; c.vrettos0@gmail.com \\ 2 Department of Materials Science and Engineering, University of Ioannina, 45110 Ioannina, Greece; \\ konstantinos.spyrou1@gmail.com \\ * Correspondence: viegeorgaki@upatras.gr; Tel.: +30-2610-996321
}

Academic Editors: Long Y. Chiang and Giuseppe Cirillo

Received: 28 January 2020; Accepted: 9 March 2020; Published: 12 March 2020

\begin{abstract}
Graphene aerogel (GA) is a lightweight, porous, environmentally friendly, 3D structured material with interesting properties, such as electrical conductivity, a high surface area, and chemical stability, which make it a powerful tool in energy storage, sensing, catalyst support, or environmental applications. However, the poor mechanical stability that often characterizes graphene aerogels is a serious obstacle for their use in such applications. Therefore, we report here the successful mechanical reinforcement of GA with carbon fibers ( $\mathrm{CFs}$ ) by combining reduced graphene oxide (rGO) and CFs in a composite material. The surfaces of the CFs were first successfully desized and enriched with epoxy groups using epichloridrine. Epoxy-functionalized CFs (epoxy-CFs) were further covered by reduced graphene oxide ( $\mathrm{rGO}$ ) nanosheets, using triethylene tetramine (TETA) as a linker. The rGO-covered CFs were finally incorporated into the GA, affording a stiff monolithic aerogel composite. The as-prepared epoxy-CF-reinforced GA was characterized by spectroscopic and microscopic techniques and showed enhanced electrical conductivity and compressive strength. The improved electrical and mechanical properties of the GA-CFs composite could be used, among other things, as electrode material or strain sensor applications.
\end{abstract}

Keywords: carbon fibers; surface treatment; grafting; graphene aerogel

\section{Introduction}

Graphene oxide $(\mathrm{GO})$ is the most common graphene derivative [1]. It is formed by the oxidative treatment of graphite and is characterized by a large amount of oxygen groups, which are spread over the graphenic surface. Carboxylates mainly at the edges, epoxy, and hydroxylates at the core provide a strong hydrophilic character and dramatically reduce the aromatic character of GO. The treatment of GO with reducing agents leads to the partial removal of the oxygen groups and the reconstruction of aromaticity, to the so-called reduced GO (rGO). Depending on the reductive treatment, rGO shows often remarkable electrical conductivity, comparable with pristine graphene. On the other hand, the removal of the majority of the oxygen groups results in the decrease in the hydrophilic character of rGO and the formation of aggregates when they are dispersed in water [2].

Hydrothermal reductive treatment of GO often leads to the formation of a stable rGO hydrogel, depending on the conditions. GO is a highly hydrophilic 2D material, which, under reductive conditions, is partially gaining its aromaticity and self-assembled in a 3D structure due to $\pi-\pi$ stacking interactions. The oxygen groups on the graphene surface, as well as the entrapped hydrophilic reducing agent, are often responsible for the entrapment of a large amount water between the rGO nanosheets, leading to the formation of stable hydrogels, which can be transformed into a graphene aerogel (GA), after water removal by a freeze-drying procedure. 
GA is a lightweight, porous, environmentally friendly 3D structured material, with electrical conductivity, chemical inertness and a high surface area [3-6]. It could be used in several applications, including in supercapacitors, lithium ion batteries, fuel or solar cells, and for environmental purposes such as water purification, gas separation, or electromagnetic interference shielding. The use of GA in most applications could be largely promoted by a significant mechanical reinforcement, which is a real challenge, taking into consideration the poor mechanical properties of GA [3-6].

On the other hand, carbon fibers (CFs) are a graphitic material that have been used widely for the reinforcement of polymers, due to their remarkable mechanical, thermal, and electrical properties as well. CFs/polymer composites have been used in applications such as in aerospace, automotive, nuclear engineering, where strength, stiffness, and lightweight materials are critical requirements [7-11]. However, their smooth and inert graphitic surface, almost free of functional groups, does not favor the adhesion with matrix polymer molecules, weakening the load that is transferred from the matrix to the CFs, and limiting the mechanical reinforcement [8,9]. Therefore, in the past decades, many research efforts have been made to modify chemically the surface of CFs, and thus to improve the interfacial adhesion in the resulting composites. However, there has been limited success up to now in the sufficient functionalization of the CF's surface. In most cases, the CF's surface is treated with strong acids, such as a nitric or nitric/sulfuric acid mixture, to introduce a limited amount of oxygen groups (mainly carboxylates) [12-14]. Carboxylates are then used as reactive sites to graft on the CF's surface coupling agents such as simple diamines, dialdehydes $[15,16]$, or, more specifically, polyphosphazenes and siloxanes [17-19]. The decoration of CFs with coupling agents increases the wettability and chemical reactivity of CFs, and this improves the adhesion between the CFs and polymer matrices. Recently, GO has been also used to modify the CF's surface, improving the interfacial properties of the composite material [20]. Furthermore, efforts have appeared in the literature that combined CFs and GA in a 3D composite structure, ideally having the properties of both components [21,22].

In the present article, we describe an effective enrichment of CFs surface with epoxy groups, and their successful incorporation in a GA, forming a 3D structured monolithic aerogel composite with improved electrical and mechanical properties. The CFs were first desized and then functionalized with epoxy groups to enhance the binding sites of the CFs, using, for the first time, epichlorohydrin. Epoxy-functionalized CFs (epoxy-CFs) were then covered by rGO nanosheets and finally incorporated successfully into the GA, during a sol-gel hydrothermal reduction process of GO. The as-prepared $\mathrm{CFs} / \mathrm{rGO}$ monolithic aerogel composite was fully characterized by spectroscopic and microscopic techniques, and finally showed enhanced electrical conductivity and compression stability. Due to these characteristics, the CFs/rGO aerogel composite could be used, among other things, as an electrode material or in strain sensor applications [23].

\section{Results and Discussion}

Initially, the CFs were desized in acetone [24] and then oxidized using an acidic treatment under ultrasonication. After this procedure, a plethora of active oxygen groups, mainly hydroxylates and carboxylates, appeared on the surface of the CFs, increasing the total surface energy and polarity, which was helpful to improve the wettability of the CFs $[25,26]$. The morphology of the CFs was studied using scanning electron microscopy (SEM), and characteristic micrographs are given in Figure 1.

The polymer removal alters the morphology and increases the roughness of the CFs' surface. The characteristic change in the roughness of CFs after desizing is indicated by atomic force microscopy (AFM) images, as shown in Figure 2. The surfaces of untreated CFs seem to be relatively neat and smooth. Few narrow grooves, distributed in parallel along the longitudinal direction of the fiber, are due to the fiber manufacture process. Compared with the untreated CFs (Figure 1a), the fiber surface becomes rougher after oxidative treatment. 

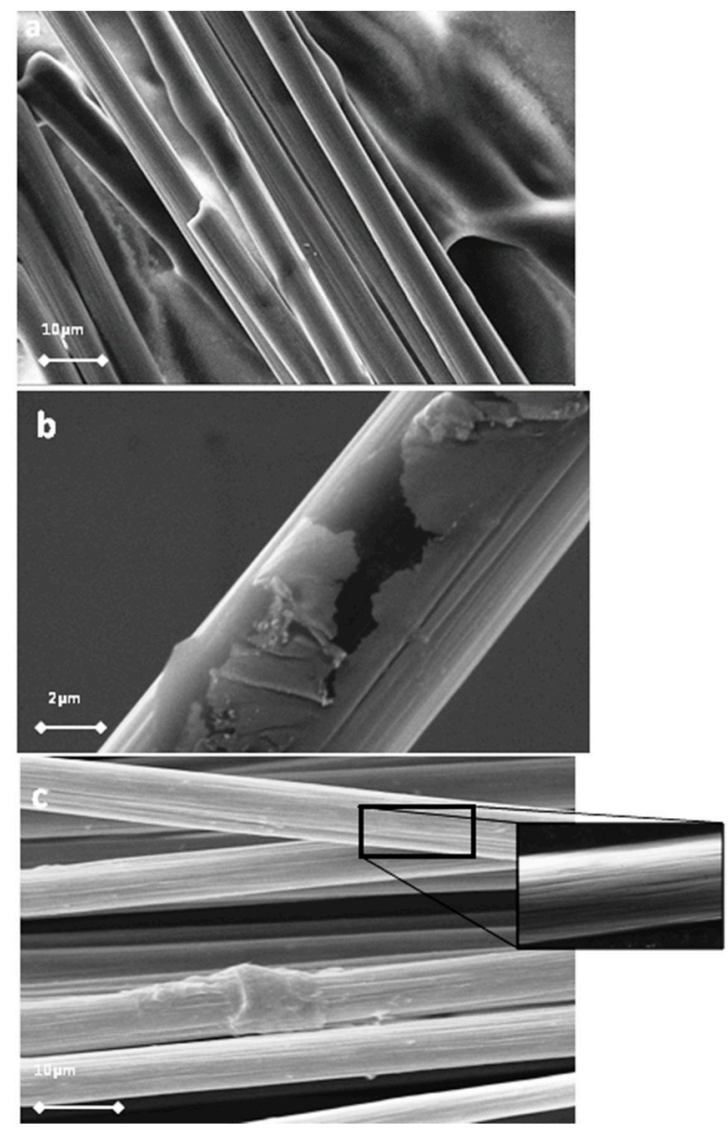

Figure 1. Scanning electron microscopy (SEM) images of carbon fibers (CFs) before (a) and after the treatment with acetone $(\mathbf{b}, \mathbf{c})$.

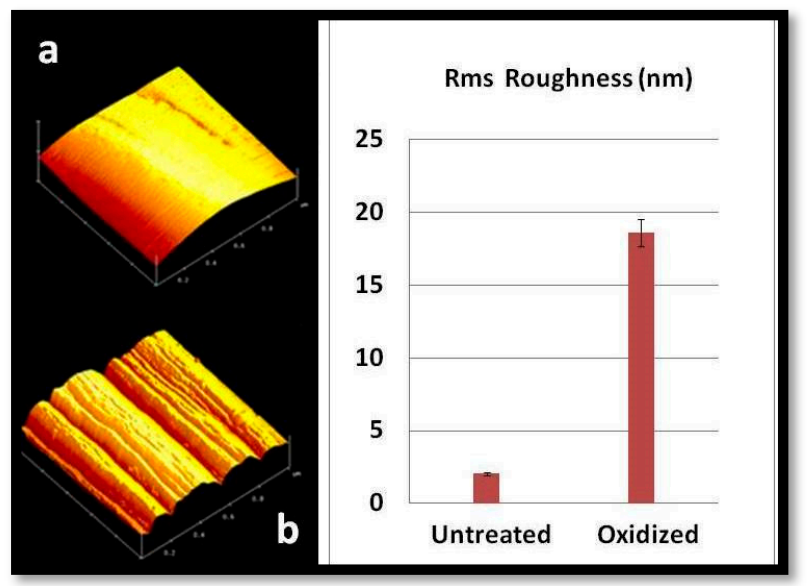

Figure 2. Atomic force microscopy (AFM) topography images of (a) untreated and (b) oxidized CFs.

Thermogravimetric and differential thermal analysis (TG-DTA) curves of pristine CFs and oxidized $\mathrm{CFs}$ are depicted in Figure 3. Pristine CFs were stable until $700{ }^{\circ} \mathrm{C}$ in the air, while, after desizing and acid treatment, oxidized CFs were decomposed much easier between 500 and $700{ }^{\circ} \mathrm{C}$ (see Figure 3). The decomposition of both the pristine and oxidized CFs was accompanied by an analogous exothermic peak, recorded in DTA as expected. Pristine CFs also indicated a $2 \%$ weight loss between 350 and $500{ }^{\circ} \mathrm{C}$, which could be attributed to the removal of the sizing agent. 

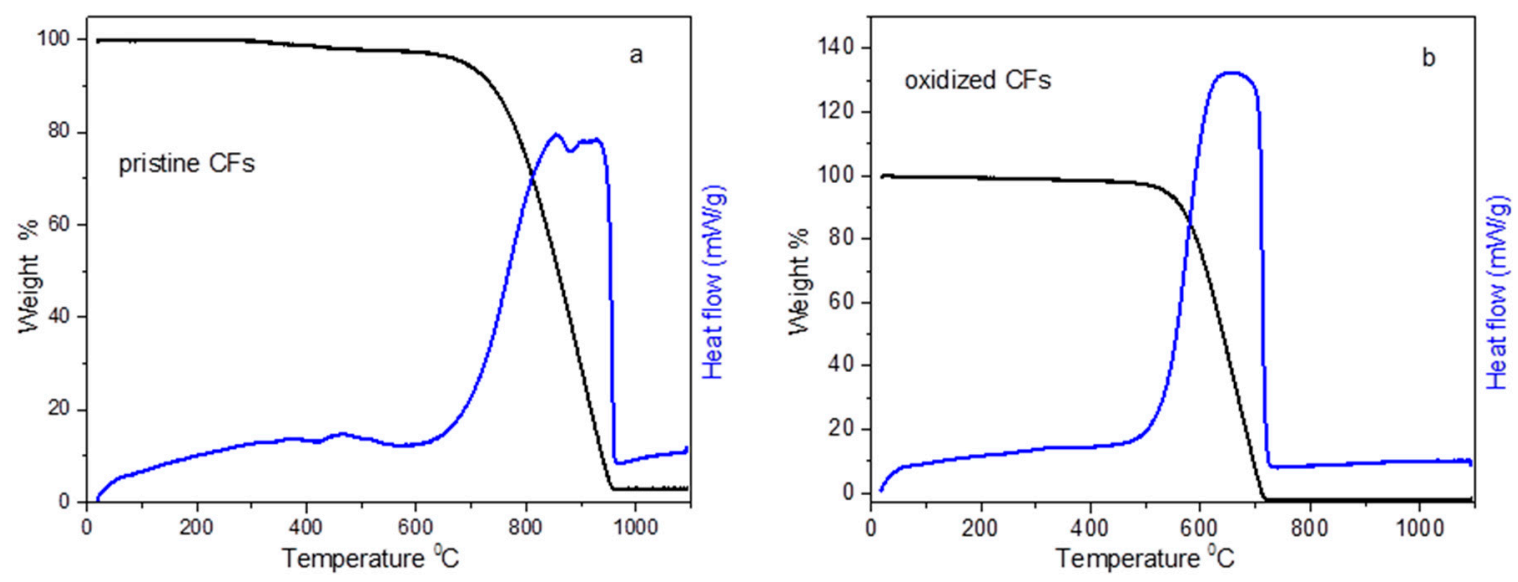

Figure 3. Thermo gravimetric analysis (TGA) and heat flow graphs of pristine (a) and oxidized CFs (b).

The Fourier-transform infrared spectrometer (FT-IR) spectrum of the oxidized CFs (see line b in Figure 4) showed a few weak but characteristic peaks due to the presence of oxygen groups on their surface, in contrast with the featureless spectrum of pristine CFs (see line a in Figure 4). The peaks at $3300\left(\mathrm{OH}\right.$ stretching vibrations) and $1033 \mathrm{~cm}^{-1}$ (C-O stretching vibrations) indicated the appearance of hydroxyl groups on the CF surface. The peaks at 1715 and $1640 \mathrm{~cm}^{-1}$ ( $\mathrm{C}=\mathrm{O}$ stretching) could be attributed to the presence of carboxylates and carbonyl groups, respectively, while peaks at 2860, 2930, and $1335 \mathrm{~cm}^{-1}$ indicate sp ${ }^{3} \mathrm{C}-\mathrm{H}$ stretching [27-29].

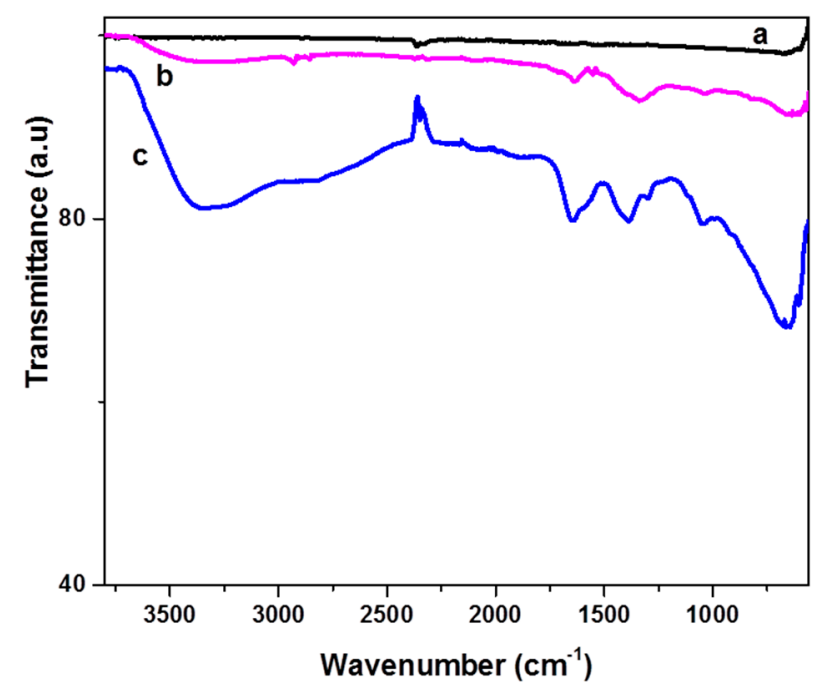

Figure 4. Fourier-transform infrared spectrometer (FT-IR) spectrum of (a) pristine CFs (b) acid treated CFs and (c) epoxy-functionalized CFs (epoxy-CFs).

Epichlorohydrin was then grafted on the CFs following mainly two different pathways. It can be added to the carboxylates or $\mathrm{OH}$ groups using the epoxy or chlorine end, respectively, as shown in Figure 5. Both pathways in the alkaline environment lead to the formation of epoxy groups on the surface of the CFs (epoxy-CFs). The FT-IR spectrum of the epoxy-CFs showed their enrichment with hydroxyl and epoxy groups, as indicated by the $\mathrm{OH}$ stretching vibration at $3330 \mathrm{~cm}^{-1}$ and $\mathrm{C}-\mathrm{O}-\mathrm{C}$, epoxy stretching vibration at 1290 and $1050 \mathrm{~cm}^{-1}$. The characteristic $\mathrm{CH}$ and $\mathrm{C}=\mathrm{C}$ vibrations at 2850 (stretching), 1400 (in plane bending), and $1647 \mathrm{~cm}^{-1}$ (stretching), respectively, also appeared (see line c in Figure 4). 

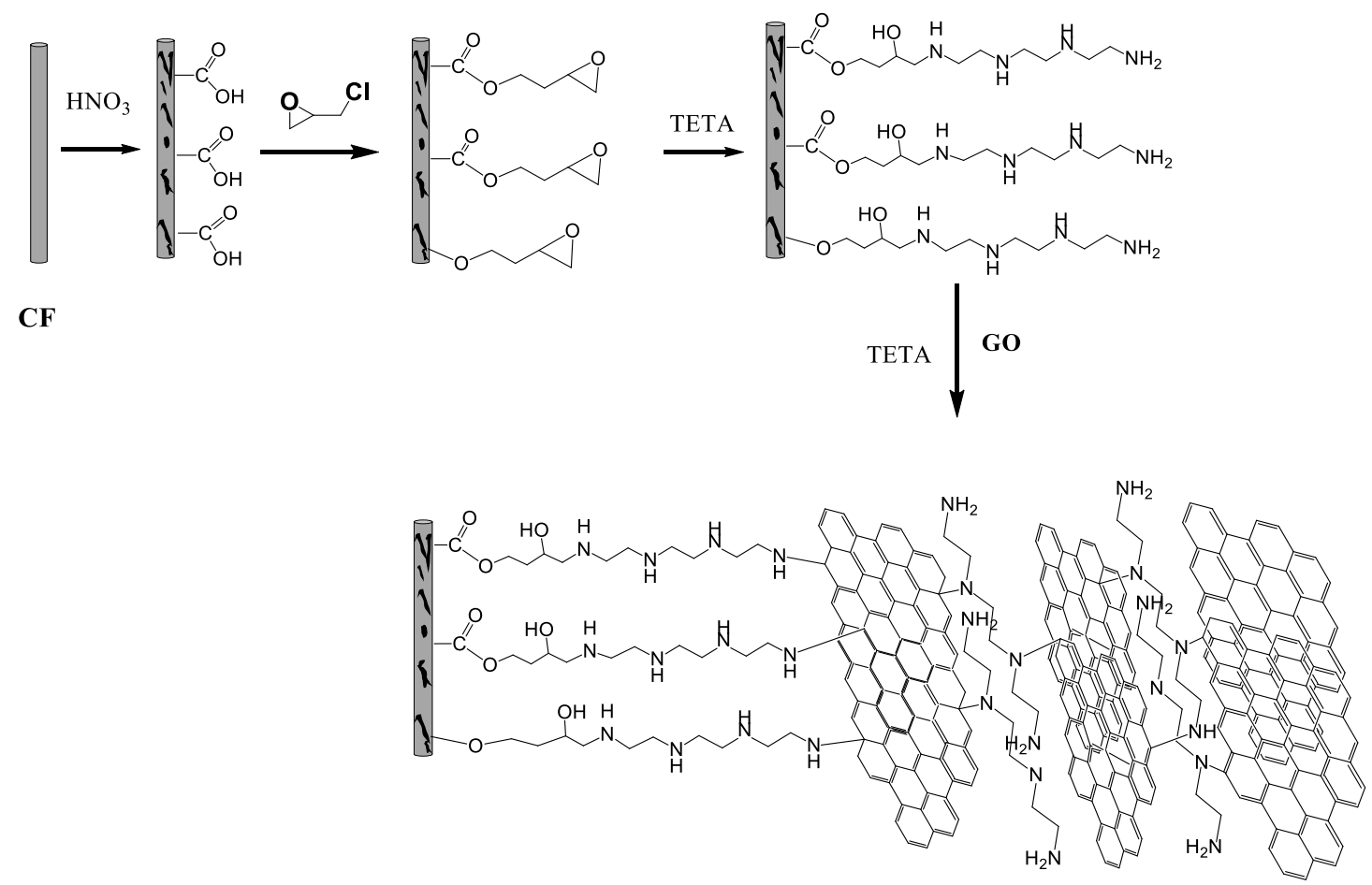

Figure 5. Schematic representation of the formation of $\mathrm{CFs} /$ reduced $\mathrm{GO}(\mathrm{rGO})$ composite aerogel.

The Raman spectra of pristine, oxidized and epoxy-CFs are shown in Figure 6. The spectrum of the CFs contains two characteristic peaks, which are assigned to the graphitic $E_{2 g} G$ mode at $\sim 1580 \mathrm{~cm}^{-1}$ and the disorder D mode at $\sim 1365 \mathrm{~cm}^{-1}$. In the spectrum of epoxy-CFs, the $\mathrm{G}$ band appeared slightly shifted to $1590 \mathrm{~cm}^{-1}$ due to the contribution of the $\mathrm{D}^{\prime}$ band at around $1610 \mathrm{~cm}^{-1}$, which is more intense after the introduction of oxygen groups on the $\mathrm{CFs}^{\prime}$ surface. The $\mathrm{I}_{\mathrm{D}} / \mathrm{I}_{\mathrm{G}}$ ratio after the reaction with epichlorohydrin was slightly increased due to the introduction of epoxy groups [30].

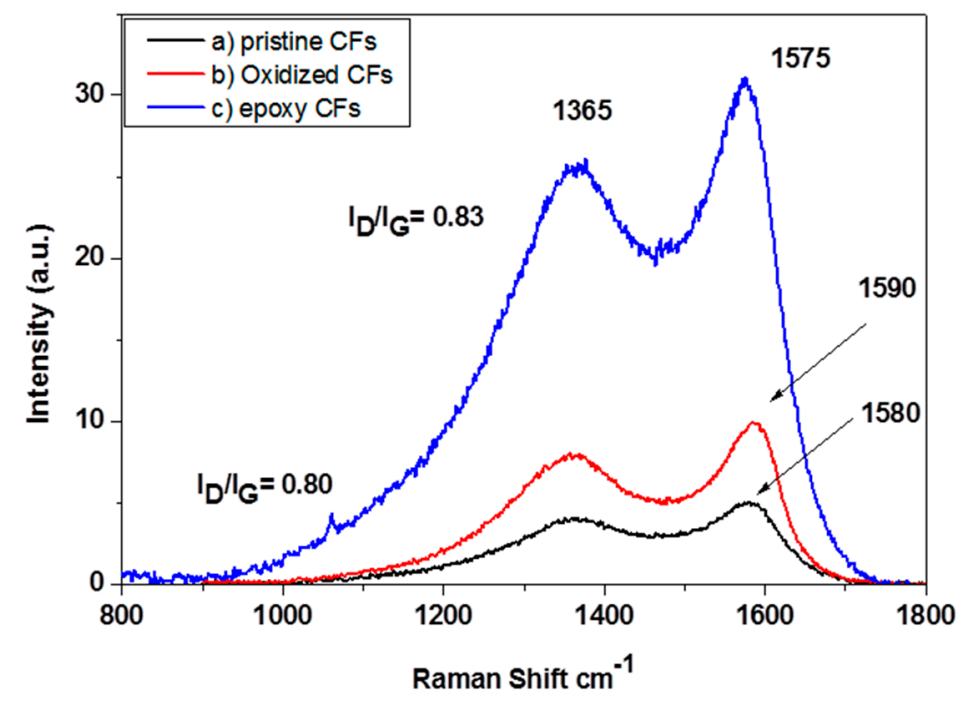

Figure 6. Raman spectra of (a) pristine (b) oxidized and (c) epoxy-CFs.

In order to identify further the functional chemical groups of the epoxy-CFs, $\mathrm{X}$-ray photoelectron spectroscopy (XPS) measurements were employed. From the C1s high resolution photoelectron spectra (Figure 7), several changes after the chemical modification were deduced. The most important information that was collected here was the reduction in the C-C frame from $80.3 \%$ for the pristine 
CFs to $29.6 \%$ for the epoxy-CFs. The peak at $286.1 \mathrm{eV}$ is increased because of the addition of C-O and also C-N bonds. The existence of these two functionalities explains the small shift from $286.1 \mathrm{eV}$, due to $\mathrm{CFs}$, to $285.8 \mathrm{eV}$. Important evidence for the successful functionalization of CFs is the significant increase in the photoelectron peak at $286.8 \mathrm{eV}$ from $3.1 \%$ to $23.0 \%$. This increase is due to the epoxy group created after the chemical functionalization $[29,31]$.
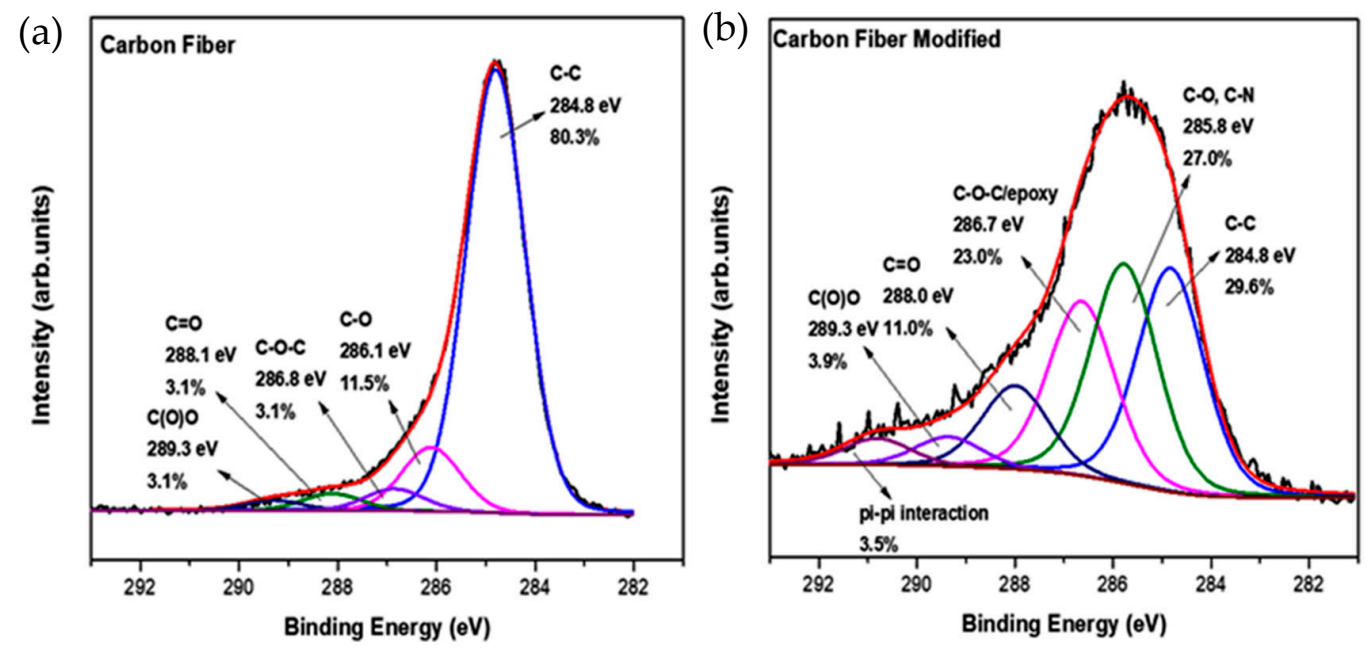

Figure 7. C1s photoelectron peak of (a) pristine CFs and (b) epoxy-CFs.

Epoxy-CFs were transferred to a diluted GO dispersion in an alkaline solution of triethylene tetramine (TETA) and heated hydrothermally at $100{ }^{\circ} \mathrm{C}$ in a sealed bottle. At this stage, the brown GO solution becomes colorless after the reaction, indicating the successful immobilization of the rGO nanosheets on the external surface of the CFs (see Figure 8a,b). The rGO-covered epoxy-CFs finally underwent a second hydrothermal treatment with a larger amount of GO dispersed in alkaline solution of TETA. Under these conditions, the GO was reduced partially and aggregated, forming a stable hydrogel (see Figure 8c). Through the nucleophilic addition to the epoxy ring opening functionalization, TETA acts here as a reducing and coupling agent on both CFs and rGO nanosheets, and thus contributes significantly to the hydrogel formation as a bridge molecule. The insertion of epoxy-CFs between the rGO nanosheets resulted in the successful incorporation of the former in the hydrogel (see Figure 8c). Finally, after freeze drying, the CFs-supported GA (CFs/rGO aerogel) was formed (see Figure 8d).

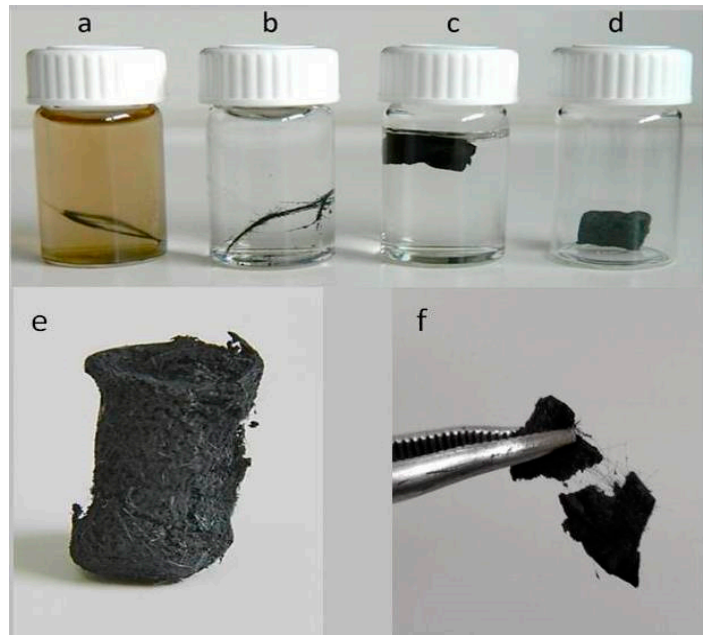

Figure 8. Photo of (a) epoxy-CFs in an alkaline solution of GO, (b) epoxy-CFs covered by the rGO nanosheets after hydrothermal heating, (c) CFs/rGO hydrogel and (d,e) aerogel, (f) CFs/rGO aerogel separated in two pieces using forcepts, where CFs are revealed from the internal. 
CFs have a mean size of $0.3-0.5 \mathrm{~cm}$ and were randomly oriented in the GA, as observed optically under a microscope. This is a consequence of the random dispersion of CFs into the mixture of the GO before the hydrothermal treatment. CFs/rGO aerogel can be also formed in a single stage hydrothermal procedure by directly dispersing epoxy-CFs in a concentrated dispersion of GO in alkaline solution of TETA. In Figure 9, several characteristic images of rGO-covered epoxy-CFs are presented (Figure 9a,b,e,f), in comparison with poorly rGO-covered pristine CFs (Figure 9c,d) that were formed when pristine CFs were used instead of epoxy-CFs. As shown in Figure 9a-d, rGO nanosheets have extensively covered the surface of the epoxy-CFs, while the absence of epoxy groups in pristine CFs leads to much less coverage by rGO, as shown in Figure 9c,d. Finally, Figure 9e,f indicate the successful incorporation of CFs in the CFs/rGO aerogel. Due to the interaction between the epoxy-CFs and GO in the presence of the TETA bridge molecules, the epoxy-CFs reinforced GA were more condensed, having a lower volume $\left(0.6 \mathrm{~cm}^{3}\right)$ than the pure $\mathrm{rGO}$ aerogel $\left(0.9 \mathrm{~cm}^{3}\right)$. In addition, taking into consideration the masses of the components and the final products, epoxy-CFs reinforced GA showed a higher density $\left(31.6 \mathrm{mg} / \mathrm{cm}^{3}\right)$ in comparison to pure $\mathrm{rGO}$ aerogel $\left(12,5 \mathrm{mg} / \mathrm{cm}^{3}\right), \mathrm{due}$ to the lower volume and the presence of CFs. The mass fraction of CFs in the CFs/rGO aerogel was estimated to be 0.31 and the volume fraction to be $5 \times 10^{-3}$.
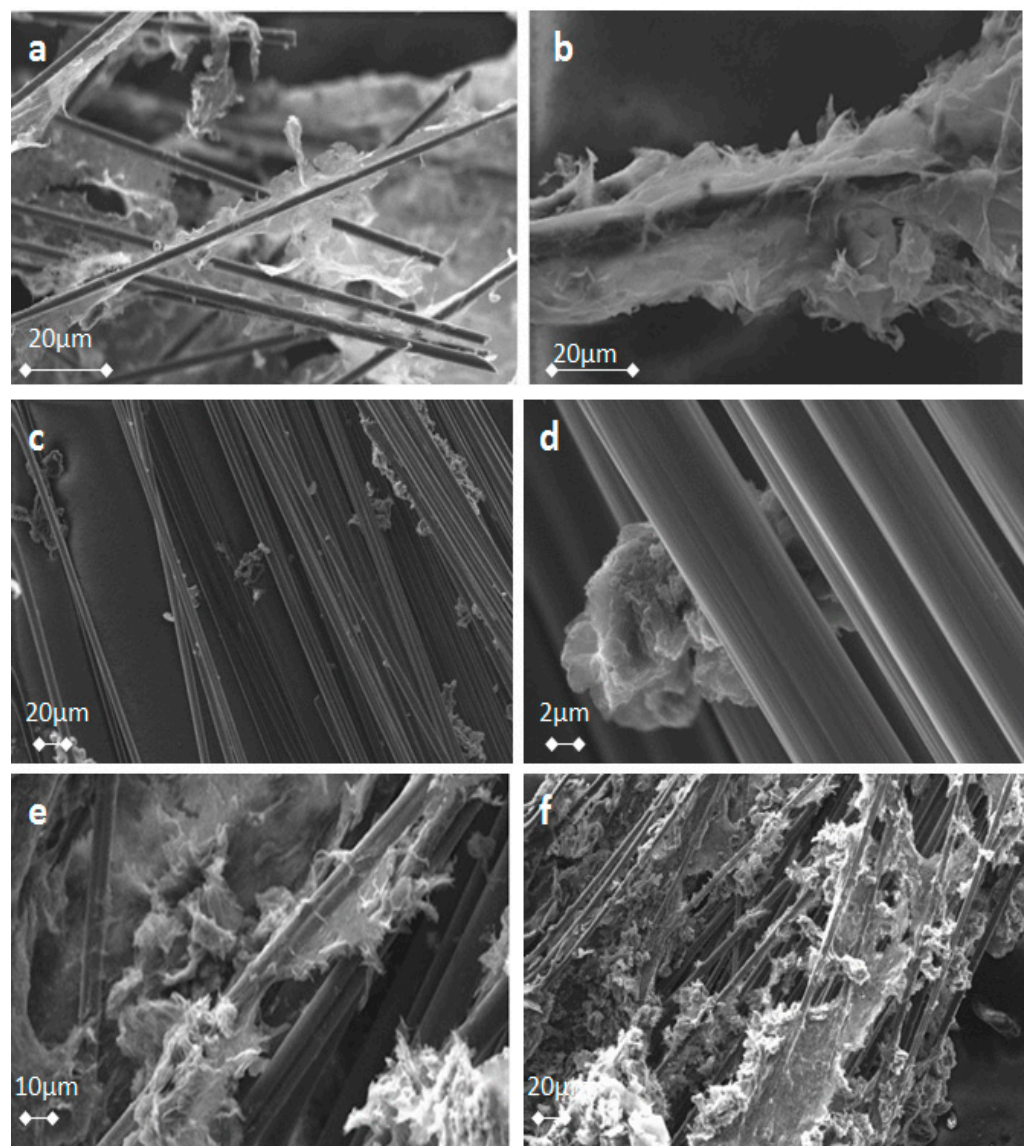

Figure 9. SEM images of epoxy-CFs $(\mathbf{a}, \mathbf{b})$ and pristine $\mathrm{CFs}(\mathbf{c}, \mathbf{d})$ covered by $\mathrm{rGO}$ nanosheets, and $\mathrm{CFs} / \mathrm{rGO}$ aerogel $(\mathbf{e}, \mathbf{f})$.

\subsection{Electrical Conductivity}

It is known that GAs are electrically conductive, due to the recovered aromaticity after the reduction of GO. CFs are also conductive, and thus the final CFs/rGO composite is highly conductive as expected (see Figure 10). In fact, the resistivity of a CFs/rGO aerogel monolith was measured to $28.8 \Omega \mathrm{m}$, while an analogous rGO aerogel monolith was measured to $129.6 \Omega \mathrm{m}$ (see Table 1 ). The samples had a cylindrical shape and the resistance was measured by adapting two electrodes at 
the upper and lower surface of the cylinders (see experimental part). It is important to note here that the orientation of the CFs in the aerogel was random and not involved with the increased conductivity of the epoxy-CFs supported GA.

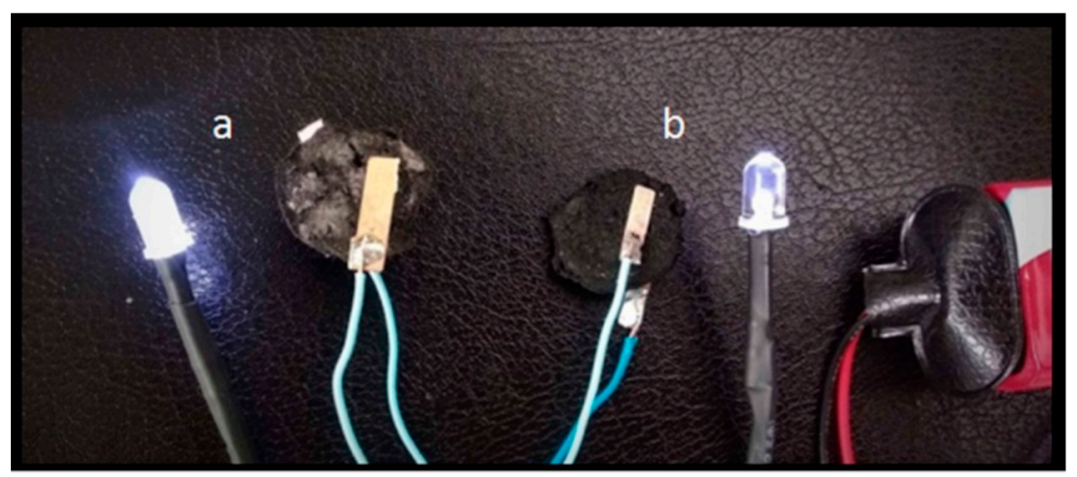

Figure 10. Electrical circuits with (a) $\mathrm{CFs} / \mathrm{rGO}$ and (b) rGO aerogel monoliths. The intense light of the LED lamp in circuit (a) indicates the increased conductivity of $\mathrm{CFs} / \mathrm{rGO}$ aerogel.

Table 1. Bulk resistance (R) and resistivity (r) of rGO and $\mathrm{CFs} / \mathrm{rGO}$ aerogels.

\begin{tabular}{ccccc}
\hline & $\mathbf{S}\left(\mathbf{m}^{\mathbf{2}}\right) \times \mathbf{1 0}^{-\mathbf{4}}$ & $\boldsymbol{l}(\mathbf{m})$ & $\mathbf{R}(\mathbf{k} \boldsymbol{\Omega})$ & $\varrho(\boldsymbol{\Omega} \mathbf{~ m})$ \\
\hline $\mathrm{rGO}$ & 1.8 & 0.005 & 3.6 & 129.6 \\
$\mathrm{CFs} / \mathrm{rGO}$ & 1.8 & 0.005 & 0.8 & 28.8 \\
\hline
\end{tabular}

\subsection{Mechanical Reinforcement}

In a recent previous article, we showed that TETA-promoted rGO aerogels can be compressed to about $50 \%$ of the initial thickness by the placement of a $50 \mathrm{~g}$ standard weight on a GA cylindrical monolith, while the rGO aerogels promoted by aromatic diamines were compressed almost elastically [32]. Here, we demonstrate that TETA-promoted GA reinforced with CFs was not compressed under the same conditions, indicating the remarkable role of CFs on the mechanical reinforcement of the CFs/rGO aerogel (see Figure 11a-c). In contrast, GA reinforced with pristine CFs by the same procedure is fragile and mechanically very unstable, leading to negative results as regards mechanical measurements. This fact indicated the crucial role of epoxy groups in the successful incorporation of CFs in the GA.

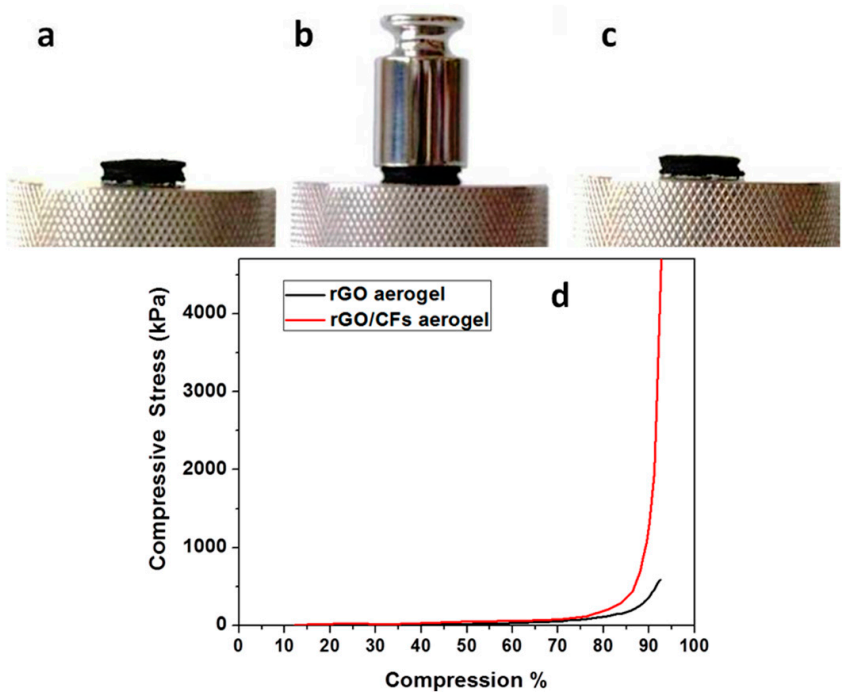

Figure 11. (Upper) $\mathrm{CFs} / \mathrm{rGO}$ cylindrical aerogel monolith before (a) during (b) and after (c) the compression, with a $50 \mathrm{~g}$ standard weight. (d) The stress/compression diagram of rGO and CFs/rGO aerogel monoliths. 
A similar conclusion was drawn by comparing the diagrams of compressive stress to compression of the rGO and the CFs/rGO aerogels (see Figure 11d). In fact, the rGO aerogel was compressed to about $90 \%$ with a $400 \mathrm{kPa}$ stress, while in the case of the CFs/rGO aerogel, a remarkably higher compressive stress-about $1300 \mathrm{kPa}$-was applied to achieve a similar compression. This 3D porous, conductive, and highly stable CFs/rGO structure could become a highly promising material for applications in lightweight conductive cables, energy storage, catalysts, and functional textiles. The unique structure of those materials paves the way to design and fabricate lightweight porous materials with high performance.

\section{Materials and Methods}

\subsection{Materials}

Commercially available CFs, T700SC (Fiber Max, Volos, Greece) were used in this work. Epichlorohydrin (Alfa Aesar, Kandel, Germany), sodium hydroxide (NaOH, Sigma-Aldrich, St Louis city, $\mathrm{MO}$, USA), ammonium hydroxide $\left(\mathrm{NH}_{3}\right.$, CARLO ERBA Reagents S.A.S., Barcelona, Spain), TETA (Sigma-Aldrich, St Louis city, MO, USA), nitric acid ( $\mathrm{HNO}_{3}, 65 \%$, CARLO ERBA Reagents S.A.S., Barcelona, Spain), and powder graphite (Sigma-Aldrich, St Louis city, MO, USA) were used without further treatment. GO was prepared in the lab, according to Staudenmaier's method [33] and the synthesis is described in detail, in ref [34].

\subsection{Characterization}

TGA/DSC measurements were performed on pristine and oxidized CFs ( 10 mg) by means of a SETARAM SETSYS Evolution 18 Analyzer (SETARAM Instrumentation, Caluire, France) with $\mathrm{Al}_{2} \mathrm{O}_{3}$ crucibles, in the range of $25-1100{ }^{\circ} \mathrm{C}$. A heating rate of $10^{\circ} \mathrm{C} / \mathrm{min}$ under air flow $(16 \mathrm{~mL} / \mathrm{min})$ while used and purging was applied well before initiating the heating ramp. Buoyancy corrections were carried out through blank measurements.

Scanning electron microscopy (SEM) was carried out on a Zeiss EVO-MA10 (Carl Zeiss Microscopy $\mathrm{GmbH}$, Jena, Germany). Infrared spectra were measured on a Fourier-transform infrared spectrometer (FT-IR) using the ATR technique on an IRTracer-100 Shimadzu spectrometer (Shimadzu Europa GmbH, Duisburg, Germany). Raman spectra were collected with a Raman system Lab-Ram HR Evolution RM (Horiba-Scientific, Kyoto, Japan) using a laser excitation line at $532 \mathrm{~nm}$ (laser diode). The laser power was $1.082 \mathrm{mV}$. All Raman parameters have been carefully controlled to avoid changes in the graphene materials. Bulk resistance was measured using a Keithley 2401 multimeter (Keithley Instruments, Solon, OH, USA), using two indium tin oxide (ITO) glass slides as electrodes that covered the upper and lower surface of the samples. Atomic force microscopy (AFM) measurements were performed in tapping mode with a multimode Nanoscope IIIa (Bruker, Billerica, MA, USA), using RTESPA-300 silicon cantilevers with a nominal tip radius $8 \mathrm{~nm}$. The values of the stress/compression diagrams of rGO and rGO/CFs aerogel monoliths were recorded with a Hounsfield H20K-W test machine (rate $1.5 \mathrm{~mm} / \mathrm{min}$, Hounsfield Test Equipment, Red Hill, England).

X-ray photoelectron spectroscopy (XPS) measurements were performed in an ultra-high vacuum at a base pressure of $2 \times 10^{-10} \mathrm{mbar}$, with a SPECS GmbH spectrometer (SPECS Surface Nano Analysis $\mathrm{GmbH}$, Berlin, Germany) equipped with a monochromatic MgKa source $(\mathrm{hv}=1253.6 \mathrm{eV})$, and a Phoibos-100 hemispherical analyzer (SPECS Surface Nano Analysis GmbH, Berlin, Germany). The spectra were collected in normal emission and the energy resolution was set to $1.16 \mathrm{eV}$ to minimize measuring time. All binding energies were referenced to the C1s core level at $284.8 \mathrm{eV}$. Spectral analysis included a Shirley background subtraction and a peak deconvolution employing mixed Gaussian-Lorentzian functions, in a least square curve-fitting program (WinSpec) developed at the Laboratoire Interdisciplinaire de Spectroscopie Electronique, University of Namur, Belgium. 


\subsection{Oxidation Treatment and Functionalization of CFs}

Some $100 \mathrm{mg}$ of $\mathrm{CFs}$ were heated in acetone for $48 \mathrm{~h}$ at $60^{\circ} \mathrm{C}$. After the drying of the desized $\mathrm{CFs}$, they were oxidized in conc. $\mathrm{HNO}_{3}$ at $100{ }^{\circ} \mathrm{C}$ for $2 \mathrm{~h}$ in a sonication bath. Subsequently, the oxidized $\mathrm{CFs}$ were washed several times with deionized water until reaching $\mathrm{pH} \sim 7$ and dried at $100{ }^{\circ} \mathrm{C}$ under vacuum. Oxidized CFs were placed in a solution of epichlorohydrin, while an ethanolic solution of $\mathrm{NaOH}$ was added slowly during refluxing $\left(95^{\circ} \mathrm{C}\right)$ for $3 \mathrm{~h}$. The epoxy-CFs were finally washed with acetone and dried under a vacuum.

\subsection{Formation of CFs/rGO Aerogel}

Epoxy-CFs $(6 \mathrm{mg})$ were placed into a concentrated solution of TETA in water $(50 \% v / v)$, at $80{ }^{\circ} \mathrm{C}$ for $24 \mathrm{~h}$. After a thorough washing, the as-prepared amine functionalized CFs (amino-CFs) were transferred in an alkaline GO dispersion ( $2 \mathrm{mg}$ of GO, $50 \mu \mathrm{L}$ conc. $\mathrm{NH}_{3}$ in $20 \mathrm{~mL} \mathrm{H}_{2} \mathrm{O}$ ) at $95^{\circ} \mathrm{C}$ for $24 \mathrm{~h}$. The as prepared $\mathrm{rGO}$ functionalized $\mathrm{CFs}$ were then placed in a dispersion of $\mathrm{GO}(10 \mathrm{mg})$ and TETA $(10 \mu \mathrm{L})$ in $20 \mathrm{~mL}$ of water, and the mixture was heated in a sealed bottle at $95^{\circ} \mathrm{C}$ for $24 \mathrm{~h}$. The resulting hydrogel was washed several times with water and lyophilized for $24 \mathrm{~h}$.

\section{Conclusions}

In this work, we demonstrated that epoxy-functionalized CFs can be successfully incorporated into GA by grafting rGO to their surface and forming a composite aerogel monolith with improved electrical and mechanical properties, due to the presence of CFs. The role of epichlorohydrin in the introduction of epoxy groups to the CFs surface was crucial, since epoxy groups are the key for the successful incorporation of CFs into rGO aerogel. The as-prepared CFs/rGO aerogel showed at least four times lower electrical resistivity than rGO aerogel, since the desized CFs function as conducting pathways within the porous structure. Despite desizing, the contribution of chemical functionalization to the surface of CFs to the mechanical properties of the final composite was also remarkable.

Author Contributions: Conceptualization, V.G. and K.V., methodology K.V., K.S., writing-original draft preparation, V.G. and K.V., K.S., writing-review and editing V.G. and K.V., supervision, V.G. All authors have read and agreed to the published version of the manuscript.

Funding: This research received no external funding.

Acknowledgments: This research is co-financed by Greece and the European Union (European Social Fund- ESF) through the Operational Programme «Human Resources Development, Education and Lifelong Learning» in the context of the project "Strengthening Human Resources Research Potential via Doctorate Research" (MIS-5000432), implemented by the State Scholarships Foundation (IK $\Upsilon$ ).

Conflicts of Interest: The authors declare no conflicts of interest.

\section{References and Note}

1. Chen, D.; Feng, H.; Li, J. Graphene Oxide: Preparation, Functionalization, and Electrochemical Applications Information. Chem. Rev. 2012, 112, 6027-6053. [CrossRef] [PubMed]

2. Pei, S.; Cheng, H.M. The reduction of graphene oxide. Carbon 2012, 50, 3210-3228. [CrossRef]

3. Hu, H.; Zhao, Z.; Wan, W.; Gogotsi, Y.; Qiu, J. Ultralight and Highly Compressible Graphene Aerogels. Adv. Mater. 2013, 25, 2219-2223. [CrossRef] [PubMed]

4. Tang, G.; Jiang, Z.G.; Li, X.; Zhang, H.B.; Dasari, A.; Yu, Z.Z. Three-dimensional graphene aerogels and their electrically conductive composites. Carbon 2014, 77, 592-599. [CrossRef]

5. Mao, J.; Iocozzia, J.; Huang, J.; Meng, K.; Lai, Y.; Lin, Z. Graphene aerogels for efficient energy storage and conversion. Energy Environ. Sci. 2018, 11, 772-799. [CrossRef]

6. Li, C.; Ding, M.; Zhang, B.; Qiao, X.; Liu, C.Y. Graphene aerogels that withstand extreme compressive stress and strain. Nanoscale 2018, 10, 18291-18299. [CrossRef]

7. Prashanth, S.; Subbaya, K.M.; Nithin, K.; Sachhidananda, S. Fiber Reinforced Composites-A Review. J. Mater. Sci. Eng. 2017, 6, 341. [CrossRef] 
8. Chen, J.; Xu, H.; Liu, C.; Mi, L.; Shen, C. The effect of double grafted interface layer on the properties of carbon fiber reinforced polyamide 66 composites. Compos. Sci. Technol. 2018, 168, 20-27. [CrossRef]

9. Koutroumanis, N.; Manikas, A.C.; Pappas, P.N.; Petropoulos, F.; Sygellou, L.; Tasis, D.; Papagelis, K.; Anagnostopoulos, G.; Galiotis, C. A novel mild method for surface treatment of carbon fibres in epoxy matrix Composites. Compos. Sci. Technol. 2018, 157, 178-184. [CrossRef]

10. Rajak, D.K.; Pagar, D.D.; Menezes, P.L.; Linul, E. Fiber-Reinforced Polymer Composites: Manufacturing, Properties, and Applications. Polymers 2019, 11, 1667. [CrossRef]

11. Anguita, J.V.; Smith, C.T.G.; Stute, T.; Funke, M.; Delkowski, M.; Silva, S.R.P. Dimensionally and environmentally ultra-stable polymer composites reinforced with carbon fibres. Nat. Mater. 2019, 19, 317-322. [CrossRef] [PubMed]

12. Tiwari, S.; Bijwe, J. Surface Treatment of Carbon Fibers-A Review. Procedia Technol. 2014, 14, 505-512. [CrossRef]

13. Dai, Z.; Shi, F.; Zhang, B.; Li, M.; Zhang, Z. Effect of sizing on carbon fiber surface properties and fibers/epoxy interfacial adhesion. Appl. Surf. Sci. 2011, 257, 6980-6985. [CrossRef]

14. Zhang, G.; Sun, S.; Yang, D.; Dodelet, J.P.; Sacher, E. The surface analytical characterization of carbon fibers functionalized by H2SO4/HNO3 treatment. Carbon 2008, 46, 196-205. [CrossRef]

15. Choi, M.H.; Jeon, B.H.; Chung, I.J. The effect of coupling agent on electrical and mechanical properties of carbon fiber/phenolic resin composites. Polymer 2000, 41, 3243-3252. [CrossRef]

16. Pittman Jr, C.U.; Wu, Z.; Jiang, W.; He, G.-R.; Wu, B.; Li, W.; Gardner, S.D. Reactivities of amine functions grafted to carbon fiber surfaces by tetraethylenepentamine. Designing interfacial bonding. Carbon 1997, 35 , 929-943. [CrossRef]

17. Becker-Staines, A.; Bremser, W.; Tröster, T. Poly(dimethylsiloxane) as Interphase in Carbon Fiber-Reinforced Epoxy Resin: Topographical Analysis and Single-Fiber Pull-Out Tests. Ind. Eng. Chem. Res. 2019, 58, 23143-23153. [CrossRef]

18. Cheng, X.; He, Z.; Luo, Y.; Zhang, X.; Lei, C. Manipulating Interfacial Strength of Polyphosphazene Functionalized Carbon Fiber Composites. Polym. Compos. 2019, 40, E1831-E1839. [CrossRef]

19. Zhang, X.; Xu, H.; Fan, X. Grafting of amine-capped cross-linked polyphosphazenes onto carbon fiber surfaces: A novel coupling agent for fiber reinforced composites. Rsc Adv. 2014, 4, 12198-12205. [CrossRef]

20. Liu, L.; Yan, F.; Li, M.; Zhang, M.; Xiao, L.; Ao, Y. Self-assembly of graphene aerogel on carbon fiber for improvement of interfacial properties with epoxy resin. Mater. Lett. 2018, 218, 44-46. [CrossRef]

21. Gao, B.; Zhang, R.; He, M.; Sun, L.; Wang, C.; Liu, L.; Zhao, L.; Cui, H.; Cao, A. Effect of a multiscale reinforcement by carbon fiber surface treatment with graphene oxide/carbon nanotubes on the mechanical properties of reinforced carbon/carbon composites. Compos. Part A Appl. Sci. Manuf. 2016, 90, 433-440. [CrossRef]

22. Keyte, J.; Pancholi, K.; Njuguna, J. Recent Developments in Graphene Oxide/Epoxy Carbon Fiber-Reinforced Composites. Front. Mater. 2019, 6, 224. [CrossRef]

23. Qin, Y.; Qu, M.; Pan, Y.; Zhang, C.; Schubert, D.W. Fabrication, characterization and modelling of triple hierarchic PET/CB/TPU composite fibres for strain sensing. Compos. Part A Appl. Sci. Manuf. 2020, 129, 105724. [CrossRef]

24. Zhao, M.; Meng, L.; Ma, L.; Ma, L.; Yang, X.; Huang, Y.; Ryu, J.E.; Shankar, A.; Li, T.; Yan, C.; et al. Layer-by-layer grafting CNTs onto carbon fibers surface for enhancing the interfacial properties of epoxy resin composites. Compos. Sci. Technol. 2018, 154, 28-36. [CrossRef]

25. Peng, Q.; Li, Y.; He, X.; Lv, H.; Hu, P.; Shang, Y.; Wang, C.; Wang, R.; Sritharan, T.; Du, S. Interfacial enhancement of carbon fiber composites by poly(amido amine) functionalization. Compos. Sci. Technol. 2013, 74, 37-42. [CrossRef]

26. Xu, H.; Zhang, X.; Liu, D.; Chun, Y.; Fan, X.; Chun. A high efficient method for introducing reactive amines onto carbonfiber surfaces using hexachlorocyclophosphazene as a new coupling agent. Appl. Surf. Sci. 2014, 320, 43-51. [CrossRef]

27. Islam, M.S.; Deng, Y.; Tong, L.; Faisal, S.N.; Roy, A.K.; Minett, A.I.; Gomes, V.G. Grafting carbon nanotubes directly onto carbon fibers for superior mechanical stability: Towards next generation aerospace composites and energy storage applications. Carbon 2016, 96, 701-710. [CrossRef] 
28. Wang, L.; Liu, N.; Guo, Z.; Wu, D.; Chen, W.; Chang, Z.; Yuan, Q.; Hui, M.; Wang, J. Nitric Acid-Treated Carbon Fibers with Enhanced Hydrophilicity for Candida tropicalis Immobilization in Xylitol Fermentation. Materials 2016, 9, 206. [CrossRef]

29. Wu, T.; Wang, G.; Dong, Q.; Qian, B.; Meng, Y.; Qiu, J. Asymmetric capacitive deionization utilizing nitric acid treated activated carbon fiber as the cathode. Electrochim. Acta 2016, 176, 426-433. [CrossRef]

30. For comparison, TGA and Raman spectra of GO material and rGO aerogels have been recorded and analyzed in our previous work in ref [32].

31. Ganguly, A.; Sharma, S.; Papakonstantinou, P.; Hamilton, J. Probing the Thermal Deoxygenation of Graphene Oxide Using High-Resolution In Situ X-ray-Based Spectroscopies. J. Phys. Chem. C 2011, 115, 17009-17019. [CrossRef]

32. Vrettos, K.; Karouta, N.; Loginos, P.; Donthula, S.; Gournis, D.; Georgakilas, V. The Role of Diamines in the Formation of Graphene Aerogels. Front. Mater. 2018, 5. [CrossRef]

33. Staudenmaier, L. Verfahren zur Darstellung der Graphitsäure. Ber. Der Dtsch. Chem. Ges. 1898, 31, $1481-1487$. [CrossRef]

34. Antonelou, A.; Sygellou, L.; Vrettos, K.; Georgakilas, V.; Yannopoulos, S.N. Efficient defect healing and ultralow sheet resistance of laser-assisted reduced graphene oxide at ambient conditions. Carbon 2018, 139, 492-499. [CrossRef]

Sample Availability: Samples of the compounds are available from the authors.

(C) 2020 by the authors. Licensee MDPI, Basel, Switzerland. This article is an open access article distributed under the terms and conditions of the Creative Commons Attribution (CC BY) license (http://creativecommons.org/licenses/by/4.0/). 